\title{
Videovalutare l'agire competente dello studente
}

\section{Videoassessing competence action's student}

\author{
Loredana Perla $^{*}$ e Viviana Vinci*^
}

\begin{abstract}
Riassunto
La videoanalisi è un oggetto di studio che vanta una consolidata tradizione. Pratica multimetodologica e area di studio interdisciplinare, essa risponde a numerosi problemi conoscitivi posti dalla complessità dei fenomeni didattici da indagare e propone strumenti e tecnologie che hanno un potenziale di descrizione, riproduzione e comprensione dei fenomeni molto alto. L'analisi video di un compito autentico costituisce un dispositivo di "supporto" per realizzare la valutazione dell'agire competente dello studente: un prototipo in grado di supportare la focalizzazione delle azioni che strutturano la competenza da valutare, per identificarne dimensioni, indicatori e livelli. Si descrive un percorso di ricerca-formazione che ha coinvolto 30 insegnanti di 11 Istituti scolastici della regione Puglia. Esito del progetto la descrizione del prototipo di videovalutazione che supporta gli insegnanti nella costruzione della rubrica valutativa e l'espressione del giudizio finale per livelli. L'attività riflessiva sollecitata attraverso l'uso del dispositivo proposto, ha avuto l'obiettivo di accompagnare gli insegnanti a riconsiderare criticamente il proprio l'agire, sollecitando lo sviluppo e il consolidamento della propria professionalità. La ricerca ha consentito di promuovere negli insegnanti un habitus di riflessione sul proprio "fare scuola" e sulle proprie strategie valutative, sul modo in cui si gestisce la mediazione tra soggetti in apprendimento, contenuti disciplinari, metodologie, strategie e dispositivi adottati.
\end{abstract}

Parole chiave: videoanalisi, valutazione, compito autentico, documentazione, sviluppo professionale

\footnotetext{
${ }^{*}$ Università degli Studi di Bari Aldo Moro.

** Università Mediterranea di Reggio Calabria.

${ }^{\wedge}$ Il contributo è frutto di un lavoro condiviso. Loredana Perla è autrice dei paragrafi 1, 3, Viviana Vinci è autrice dei paragrafi 2, 4. Tutti gli autori hanno contributo alla revisione del manoscritto nella sua versione finale.
}

Excellence and Innovation in Learning and Teaching (ISSNe 2499-507X), 2021, 1

Doi: 10.3280/exioa1-2021oa12075 


\begin{abstract}
Video analysis is a field of study with a long well-established tradition. As a multi-methodological practice and an area of interdisciplinary study, it responds to several cognitive problems posed by the complexity of educational phenomena to be investigated and offers tools and technologies that have a very high potential to describe, reproduce and understand phenomena. The video analysis of an authentic task constitutes a "support" device to carry out the assessment of the student's competent action: a prototype able to detect the focus on actions that structure the competence to be assessed, in order to identify its dimensions, indicators and levels. A research-training itinerary is described, which involved 30 teachers from 11 schools in the region of Apulia. The outcome of the project is the description of the video assessment prototype, which supports the teachers in the construction of the assessment rubric and the final judgement. The reflective activity stimulated in the teachers, through the use of the proposed device, had the aim of accompanying them to critically reconsider their actions, encouraging the development and consolidation of their professionalism. The research has therefore made it possible to promote in teachers - through the systematic use of a video-evaluation device - a habitus of reflection on their own "doing school" and, more specifically, on their assessment strategies, on the way in which they manage the mediation between learners, disciplinary contents, methodologies, strategies and devices adopted. Key Words: video analysis, assessment, authentic task, documentation, professional development
\end{abstract}

\title{
1. La videoanalisi: framework teorico
}

La videoanalisi è un oggetto di studio che vanta una consolidata tradizione. Quest'ultima, iniziata con le prime esperienze di microteaching all'Università di Stanford (Allen, 1966), ha avuto un seguito con gli studi sull'uso dei video per la teacher effectiveness (Orme, 1966) e, più recentemente, per la formazione iniziale e in servizio degli insegnanti (Santagata, Guarino, 2011; Rossi, 2017).

Definito anche un amplificatore pedagogico (Cescato, Bove, Braga, 2015) in grado di facilitare l'osservazione delle attività e di ridurre il divario tra teoria e pratica, il video è un mediatore didattico versatile: un testo mediale, caratterizzato dalle variabili - forma, significato, funzione - che si pone rispetto al gruppo come medium comunicativo-dialogico-relazionale nella costruzione condivisa dei significati. Diversi sono gli obiettivi per i quali esso può essere utilizzato in percorsi di formazione e ricerca: l'apprendimento dalla pratica; il miglioramento delle conoscenze disciplinari degli insegnanti, il miglioramento 
dell'osservazione, la riflessione sulla propria visione professionale, il confronto fra pari, la documentazione di prodotti e processi didattici.

L'utilizzo del video si colloca nel framework delle tecniche di "clinica" dell'attività professionale messe a punto in ambiente francofono all'interno di percorsi di ricerca-formazione di tipo collaborativo. In tali modelli - il cui frame è l'analisi di pratiche (Altet, 2002, 2003) - l'uso del video promuove una postura interrogante e un pensiero critico rispetto all'agito didattico, quindi contribuisce a promuovere lo sviluppo professionale del docente.

Come ben enfatizzano Mangione e Rosa (2017), il video può essere un valido supporto nella competenza di professional vision del docente, ossia la capacità del docente di cogliere le dinamiche che possono istaurarsi all'interno del contesto educativo e di individuare le scelte strategiche in grado di massimizzare le opportunità di apprendimento per gli studenti attraverso la rimodulazione dell'azione didattica: il dispositivo del video, infatti, mette il docente nella condizione di predire gli impatti di una strategia didattica rispetto agli obiettivi; consente di catturare la complessa realtà scolastica in modo autentico, mettendo in evidenza le azioni che si succedono nello stesso luogo contemporaneamente; consente, per un verso, l'immersione e il coinvolgimento dell'osservatore (Goldman, 2007) e, per altro verso, un distanziamento riflessivo, dato dal fatto di fornire una registrazione permanente, che può essere visualizzata, messa in pausa o riguardata più volte, per riflettere su situazioni anche da prospettive diverse e "in differita". La post-riproduzione e il rallentamento delle immagini consente di analizzare, descrivere e comprendere meglio gli eventi. La videoregistrazione "lascia una traccia" dell'esperienza, della dimensione verbale dei discorsi e di quella visiva dei comportamenti.

Il video può essere utilizzato come strumento epistemologico utile all'analisi e alla riflessione su contesti e azioni: il materiale audiovisivo se analizzato in partnership col ricercatore può divenire un ottimo punto di partenza per favorire processi non solo di carattere esplicitativo ma anche auto-valutativo (Perla, Schiavone, 2014, 2017). In questa direzione occorre evidenziare che la video-analisi, pratica multimetodologica e area di studio interdisciplinare, risponde a numerosi problemi conoscitivi posti dalla complessità dei fenomeni didattici da indagare e propone strumenti e tecnologie che hanno un potenziale di descrizione, riproduzione e comprensione dei fenomeni molto alto, non solo per i ricercatori ma anche per chi quei fenomeni li vive da protagonista: gli insegnanti.

Come già sottolineato altrove (Perla, Schiavone, 2017), l'uso dei video sollecita la dimensione metacognitiva e riflessiva sulla pratica didattica. In questa direzione diverse sono le variabili sulle quali far concentrare l'osservazione nelle fasi di accompagnamento formativo all'analisi-video: 
- le variabili di finalizzazione, inerenti agli scopi dell'insegnamento (di socializzazione e metacognizione);

- le variabili tecniche, coincidenti con $i$ "gesti professionali" del mestiere e le componenti di progettazione, valutazione, organizzazione;

- le variabili comunicative mediate dal linguaggio verbale e non;

- le variabili di "contestualizzazione" relative alla cultura di scuola;

- le variabili relazionali, che includono tutte le pratiche di magistralità educativa: accompagnamento, cura, personalizzazione, ascolto, clinicità.

A queste si aggiungono le variabili dell'apprendimento dell'allievo, analizzabili attraverso domande focalizzate sui modi dell'apprendere degli studenti e su interrogativi posti riguardo i progressi o i regressi che è possibile evidenziare dall'analisi dei video. Ciascuna di queste variabili si presta ad essere analizzata attraverso protocolli di osservazione che agevolino la riflessione dell'insegnante sui modi in cui il suo agire didattico potrebbe essere migliorato.

La nostra ricerca si è concentrata sulle variabili inerenti l'agire valutativo del docente (Galliani, 2015). Più specificamente, la consegna data agli insegnanti è stata quella di realizzare e analizzare un video su un compito autentico e di co-costruire, a partire dall'analisi delle esperienze di videoanalisi, un dispositivo di "supporto" per realizzare la valutazione dell'agire competente dello studente: un prototipo in grado di supportare la focalizzazione delle azioni che strutturano la competenza da valutare.

La letteratura che interconnette videoanalisi e valutazione è esigua.

Su tale ambito specifico si richiamano:

- le riflessioni di Ackermans, Rusman, Brand-Gruwel e Specht (2017) sull'efficacia delle Video Enhanced Rubrics (VER) nella valutazione autentica, ossia l'uso combinato di video e di rubriche testuali statiche, in grado di favorire lo sviluppo di modelli mentali da parte degli studenti, la qualità del feedback e la padronanza di abilità complesse;

- lo studio di Henderson e Phillips (2015) sul video-based feedback, ossia sul feedback di valutazione basato su video, considerato da insegnanti e studenti uno strumento efficace in quanto maggiormente personalizzato, motivante, chiaro, dettagliato e non ambiguo;

- il progetto LLP Comenius FAMT\&L, Formative Assessment in Mathematics for Teaching and Learning, che ha avuto come principale finalità la realizzazione di un corso di formazione per i docenti sulla valutazione formativa 
focalizzata sull'analisi di filmati di momenti di valutazione formativa realizzati in aula (Franchini, Salvisberg, Sbaragli, 2016).

\section{Disegno della ricerca}

L'itinerario progettuale dal titolo "L'innovazione didattica per/con lo studente", nato per supportare le scuole nel settore della ricerca educativa e della valutazione degli apprendimenti e di sistema, è stato promosso dal Dipartimento For.Psi.Com. dell'Università di Bari e dal gruppo di ricerca DidaSco Didattiche Scolastiche (coordinamento: L. Perla) in collaborazione con l'USRPuglia.

Il progetto ha coinvolto 30 insegnanti di 11 Istituti scolastici della regione Puglia $^{1}$ in un itinerario progettuale di ricerca-formazione centrato sulla co-costruzione e sperimentazione di dispositivi documentali innovativi ed efficaci nel supportare gli insegnanti nella valutazione degli apprendimenti degli studenti, in particolare nell'analisi e valutazione dell'agire competente: un agire che necessita di un'attività di riflessione, osservazione e analisi del processo e delle "tracce" di questo agire in situazione. Più specificatamente l'itinerario progettuale ha inteso focalizzare l'attenzione sul ruolo della videoanalisi nella valutazione.

Gli obiettivi della ricerca-formazione sono stati molteplici:

- strutturare un dispositivo di video-analisi dell'azione che sia di supporto, per gli insegnanti, nella valutazione delle competenze degli allievi nei compiti autentici;

- creare uno spazio virtuale di riflessione sulle pratiche documentali valutative all'interno della rete, a partire dalla condivisione e dall'analisi dei documenti (format, schede, procedure) utilizzati per valutare gli apprendimenti degli studenti;

- promuovere una postura riflessiva che supporti docenti e dirigenti nell'analisi della propria pratica educativa per lo sviluppo professionale;

- consapevolizzare i docenti sul nesso fra valutazione autentica e documentazione;

- affinare le competenze valutative e documentali dei docenti attraverso un percorso di professionalizzazione in servizio - formalizzato e replicabile per le scuole del primo ciclo.

L'itinerario di ricerca - progettato già nel 2016/2017 sulla base degli esiti del progetto "La storia e la scuola. Senso e metodi di un insegnamento", in cui

\footnotetext{
${ }^{1}$ Gli insegnanti coinvolti nella ricerca sono di scuola dell'infanzia, primaria e secondaria di I grado. La scuola capofila è l'Istituto “Vico-De Carolis" di Taranto (Dirigente: E. Scalera).
} 
è stata sperimentata un'attività di videoricerca di case-lectio di storia (Perla, Schiavone 2017) - è stato articolato in 4 fasi, svoltesi in 20 ore complessive di formazione in presenza suddivise in 5 incontri, alternati da attività di formazione a distanza su piattaforma e-learning. L'attivazione della community online è stata preziosa in quanto ha permesso ai docenti di condividere articoli ed esperienze, oltre che di inserire contenuti didattici destrutturati (testi, pdf, foto, video, verifiche).

\begin{tabular}{|c|c|c|c|}
\hline $\begin{array}{l}\text { Organizzazione } \\
\text { of compito autentico } \\
\text { J Focalizzazione } \\
\text { competenza/e da } \\
\text { valutare e } \\
\text { dimensioni } \\
\text { Realizzazione del } \\
\text { video centrato sul } \\
\text { compito autentico }\end{array}$ & $\begin{array}{l}\text { N Analisi del } \\
\text { o costrutto di } \\
\mathbb{E} \text { videovalutazione } \\
\text { I } \\
\text { Elaborazione di } \\
\text { una griglia di } \\
\text { analisi di compiti } \\
\text { autentici } \\
\text { Analisi video da } \\
\text { parte dei docenti e } \\
\text { caricamento dei } \\
\text { materiali su } \\
\text { piattaforma }\end{array}$ & $\begin{array}{l}\text { Analisi del } \\
\text { materiale su } \\
\text { mpiattaforma da } \\
\text { of parte del gruppo di } \\
\text { ricerca } \\
\text { Analisi delle } \\
\text { pratiche valutative } \\
\text { degli insegnanti e } \\
\text { dei dispositivi } \\
\text { documentali } \\
\text { Restituzione e } \\
\text { negoziazione }\end{array}$ & $\begin{array}{l}\checkmark \text { Elaborazione di un } \\
\text { prototipo di } \\
\widetilde{J} \text { videovalutazione } \\
\widetilde{J} \text { per migliorare le } \\
\text { prassi di } \\
\text { valutazione per } \\
\text { competenze degli } \\
\text { insegnanti }\end{array}$ \\
\hline
\end{tabular}

Fig. 1 - Articolazione delle fasi del progetto

La prima fase ha previsto l'organizzazione, da parte dei docenti, di un compito autentico con la focalizzazione della/e competenza/e e delle relative dimensioni da valutare. Per compito autentico intendiamo una situazione-problema simile a quelle vissute nella vita reale, che permette allo studente di sviluppare e acquisire competenze, che lo ponga in relazione con l'oggetto culturale da apprendere ${ }^{2}$ : si tratta di "compiti significativi, che consentano allo studente di sperimentare e fare la scoperta personale del sapere, di rapportarsi ad esso con uno spirito curioso, condividendo con gli altri questa esperienza; di acquisire, in tal modo, un sapere significativo, ossia riconosciuto come 'importante' o necessario dal soggetto, per destreggiarsi all'interno di una determinata situazione problematica" (Grion, Aquario, Restiglian, 2019, p. 94).

\footnotetext{
2 Il "compito autentico" è stato ampiamente discusso in letteratura, sia a livello nazionale che internazionale (si richiamano, fra tanti riferimenti: Herrington, Reeves, Oliver, 2014; Grion, Aquario, Restiglian, 2019). Fra le caratteristiche distintive dei contesti in cui si realizza il compito autentico richiamiamo, oltre all'essere strettamente connessi a situazioni di vita reale, anche: la complessità della situazione, che non è predefinita e che richiede un certo periodo di tempo per essere affrontata; l'accesso a forme di 'modeling', ossia di contatto con esperti in grado di offrire esempi di buone pratiche per risolvere la situazione problema, ma anche di coaching e scaffolding; la possibilità di favorire processi di conoscenza collaborativa, valorizzante molteplici prospettive interpretative e processi riflessivi.
} 
Gli insegnanti della rete di progetto sono stati sollecitati a progettare compiti autentici, situazioni-problema capaci di attivare la capacità degli studenti di utilizzare le conoscenze in modo efficace e adeguato alla realtà.

La durata del compito, comprensivo delle fasi preparatorie, è stata molto variabile anche in virtù della specificità dei target (si tratta di insegnanti sia di scuola dell'infanzia, che di scuola primaria e secondaria di I grado): al di là della diversa tempistica di realizzazione del compito, i docenti sono stati sollecitati a videoregistrare una fase di esecuzione dello stesso della durata massima di 20 minuti. La fase del compito autentico da videoregistrare è stata scelta autonomamente dai docenti, che hanno privilegiato i momenti centrali (soprattutto di interazione fra pari degli studenti) e/o finali, di presentazione/discussione del prodotto o della risoluzione della situazione problema. Gli studenti sono stati ripresi con videocamera fissa in dotazione nelle scuole partner; i video sono stati poi scaricati dalle/dai docenti referenti del progetto e caricati su piattaforma e-learning, previa autorizzazione dei genitori ${ }^{3}$.

Nella seconda fase, dopo un approfondimento del costrutto teorico della videoanalisi, i docenti sono stati sollecitati ad elaborare - sulla base di alcune schede stimolo di partenza (cfr. tab. 1) - una griglia di analisi dei compiti autentici realizzati e videoregistrati. Le schede di analisi co-elaborate da insegnanti e ricercatori sono costituite da una sezione più descrittiva dell'azione (tipologia del compito autentico, azioni dell'alunno e dell'insegnante, modi di rappresentazione del contenuto, mediatori ed organizzazione del setting) e da una sezione più analitica, strutturata a mo' di rubrica ${ }^{4}$, con identificazione, per ogni dimensione, degli indicatori e dei livelli di padronanza attesi.

Utilizzando la scheda di analisi, i docenti hanno analizzato i video e caricato su piattaforma e-learning sia i file video che le schede adattate al compito.

Nella terza fase, il gruppo di ricerca ha analizzato i materiali caricati dai docenti su piattaforma e verificato il grado di coerenza fra il video registrato e l'ipotesi di scheda di analisi, al fine di congedare un "prototipo" di scheda di videovalutazione. Gli esiti dell'analisi delle pratiche valutative degli insegnanti in classe e dei dispositivi documentali sono stati restituiti ai docenti, attivando una negoziazione triangolata fra ricercatori e insegnanti, con l'attivazione di un setting riflessivo. L'obiettivo di questa fase è stato quello di consentire agli insegnanti di descrivere e analizzare la multidimensionalità della pratica didattica agita in classe in partnership con i ricercatori.

\footnotetext{
${ }^{3}$ Si precisa che, prima dell'avvio delle attività, le/i docenti hanno richiesto a tutti i genitori degli studenti coinvolti nel progetto di firmare una liberatoria per la tutela della privacy dei minori nelle fotografie e riprese video.

${ }^{4}$ Nella costruzione della scheda si è fatto riferimento alle componenti che Castoldi (2016) individua nella costruzione della rubrica valutativa: dimensioni, criteri, indicatori, livelli, àncore.
} 


\begin{tabular}{|c|c|c|c|c|c|}
\hline \multirow{3}{*}{$\begin{array}{l}\text { SEZIONE A } \\
\text { DESCRIT- } \\
\text { TIVA } \\
\text { ANALISI } \\
\text { VIDEO } \\
\text { Compito auten- } \\
\text { tico da osser- } \\
\text { vare e valutare }\end{array}$} & \multicolumn{5}{|c|}{ SEZIONE B - ANALITICA E VALUTATIVA } \\
\hline & \multirow{2}{*}{$\begin{array}{l}\text { Dimensioni } \\
\text { di realizza- } \\
\text { zione del } \\
\text { compito au- } \\
\text { tentico }\end{array}$} & \multirow[t]{2}{*}{ Indicatore } & \multicolumn{3}{|c|}{ Livelli attesi di padronanza } \\
\hline & & & 1. Novizio & $\begin{array}{l}\text { 2. Apprendi- } \\
\text { sta }\end{array}$ & 3. Esperto \\
\hline \multirow{5}{*}{$\begin{array}{l}\text { Descrizione } \\
\text { complessiva } \\
\text { dell'azione } \\
(10-15 \text { minuti) } \\
\text { - Tipologia di } \\
\text { compito auten- } \\
\text { tico } \\
\text { - Cosa fa } \\
\text { l'alunno? } \\
\text { - Cosa fa l'in- } \\
\text { segnante? } \\
\text { - Quali sono i } \\
\text { modi di rappre- } \\
\text { sentazione del } \\
\text { contenuto? } \\
\text { - Quali media- } \\
\text { tori sono usati? } \\
\text { - Come è orga- } \\
\text { nizzato il set- } \\
\text { ting? }\end{array}$} & $\begin{array}{l}\text { Padronanza } \\
\text { del contenuto } \\
\text { e recupero } \\
\text { delle cono- } \\
\text { scenze }\end{array}$ & $\begin{array}{l}\text { Saper recu- } \\
\text { perare in- } \\
\text { formazioni } \\
\text { e cono- } \\
\text { scenze pre- } \\
\text { gresse }\end{array}$ & $\begin{array}{l}\text { Lo studente ha } \\
\text { difficoltà nel } \\
\text { recuperare au- } \\
\text { tonomamente } \\
\text { le conoscenze } \\
\text { ecc... }\end{array}$ & $\begin{array}{l}\text { Lo studente } \\
\text { recupera le } \\
\text { informazioni } \\
\text { pregresse ecc. }\end{array}$ & $\begin{array}{l}\text { Lo studente utilizza } \\
\text { con piena padronanza } \\
\text { tutte le informazioni } \\
\text { acquisite ecc. }\end{array}$ \\
\hline & $\begin{array}{l}\text { Adeguatezza } \\
\text { del registro } \\
\text { al livello co- } \\
\text { municativo }\end{array}$ & $\begin{array}{l}\text { Saper mo- } \\
\text { dulare il lin- } \\
\text { guaggio }\end{array}$ & $\begin{array}{l}\text { 区 } \\
\text { Lo studente } \\
\text { utilizza un vo- } \\
\text { cabolario ri- } \\
\text { stretto ecc. }\end{array}$ & $\begin{array}{l}\text { Lo studente } \\
\text { riconosce ed } \\
\text { utilizza di- } \\
\text { versi registri } \\
\text { comunicativi } \\
\text { ecc. }\end{array}$ & $\begin{array}{l}\text { Lo studente sa modu- } \\
\text { lare in maniera pien-- } \\
\text { mente efficace il lin- } \\
\text { guaggio tenendo } \\
\text { conto del contesto e } \\
\text { dei destinatari ecc. }\end{array}$ \\
\hline & & & & 可 & \\
\hline & $\begin{array}{l}\text { Dimensione } \\
\text { della compe- } \\
\text { tenza... }\end{array}$ & $\begin{array}{l}\text { Indicatore } \\
\text { (che mi per- } \\
\text { mette di os- }\end{array}$ & $\begin{array}{l}\text { Descrizione } \\
\text { delle àncore... }\end{array}$ & \begin{tabular}{l}
\multicolumn{2}{l}{ Descrizione } \\
delle àn- \\
core...
\end{tabular} & $\begin{array}{l}\text { Descrizione delle àn- } \\
\text { core... }\end{array}$ \\
\hline & & $\begin{array}{ll}\text { servare } & \text { e } \\
\text { valutare) } & \end{array}$ & & 凶 & \\
\hline
\end{tabular}

Nella quarta e ultima fase, è stato elaborato un prototipo di videovalutazione per migliorare le prassi di valutazione per competenze degli insegnanti.

\section{Il prototipo di videovalutazione}

Il dispositivo ha consentito di attivare, con i 30 insegnanti coinvolti delle 11 scuole, un'analisi sistematica del processo di insegnamento/apprendimento al fine di agevolare l'identificazione dei processi mediatori (Damiano, 2013) finalizzati a suscitare la competenza. Analizzare i processi mediatori finalizzati allo sviluppo delle competenze dello studente significa esplicitare il "come si insegna e apprende per competenze a scuola" illuminando le variabili implicite ed esplicite sottese al processo: quali i tempi e gli spazi dedicati, quali sono $i$ contenuti scelti dagli insegnanti, quali i mediatori privilegiati, quali le azioni degli studenti.

Più specificatamente gli insegnanti sono stati guidati - attraverso tale dispositivo - a un' analisi integrata dei tre vertici del triangolo pedagogico (Damiano, 2013): 
- l'insegnante. L'"agire docente" è estremamente complesso: dietro a un obiettivo che può sembrare semplice - far sì che gli studenti imparino - si nascondono molteplici conoscenze, abilità e competenze che gli insegnanti dovrebbero possedere per svolgere il loro lavoro in modo efficace. Per esempio, essi devono conoscere la materia che insegnano, le strategie pedagogiche che facilitano l'apprendimento in generale e l'apprendimento specifico di quella materia, le rappresentazioni utili a illustrare concetti particolarmente difficili, il livello di sviluppo cognitivo ed emotivo degli alunni, il curriculum relativo al livello scolare della materia che insegnano, il libro di testo adottato e software didattici particolarmente utili;

- gli studenti. A partire da un'attenta analisi del processo di apprendimento dei loro alunni, gli insegnanti possono approfondire le conoscenze delle rappresentazioni che più facilitano l'apprendimento, dei modi in cui gli alunni tendono a capire certi concetti matematici, delle difficoltà ed errori più comuni e delle strategie pedagogiche che appaiono particolarmente efficaci per certi livelli scolari.

- la/le disciplina/e analizzata/e nelle loro interconnessioni e nei metodi specifici.

La scheda di analisi video è composta da tre assi (cfr. tab. 2) e a ciascuna di questi tre assi è stata associata una matrice di analisi al cui interno sono state individuate degli indicatori di analisi specifici, un'area commento (indicata per ciascun indicatore, in cui inserire le osservazioni rilevate nel corso della visione del video) e un' appendice contenente i descrittori associati a ciascun indicatore:

\begin{tabular}{|c|c|c|}
\hline Asse 1 Agire insegnante & $\begin{array}{c}\text { Asse } 2 \\
\text { Agire studente }\end{array}$ & Asse 3 La mediazione \\
\hline $\begin{array}{l}\text { Area 1a - Organizzazione spazio- } \\
\text { tempo classe } \\
\text { Indicatori } \\
\text { - Gestione "spazio" docente } \\
\text { - Gestione tempo docente }\end{array}$ & $\begin{array}{l}\text { Area } 5 \text { - Il "fare” degli } \\
\text { studenti } \\
\text { Indicatori } \\
\text { - Gestione "spazio" } \\
\text { alunni } \\
\text { - Gestione tempo } \\
\text { alunni } \\
\text { - Relazioni in aula } \\
\text { - Partecipazione degli } \\
\text { studenti } \\
\text { - Risposte degli stu- } \\
\text { denti }\end{array}$ & $\begin{array}{l}\text { Area } \mathbf{6} \text { - Analisi dei conte- } \\
\text { nuti e dei metodi } \\
\text { Indicatori } \\
\text { - Qualità dei contenuti pro- } \\
\text { posti } \\
\text { - Originalità dei contenuti } \\
\text { - Adeguatezza dei richiami } \\
\quad \text { alle fonti } \\
\text { - Sviluppo argomentativo del } \\
\quad \text { discorso } \\
\text { - Capacità del racconto } \\
\text { - Apertura all'interdiscipli- } \\
\text { narità } \\
\text { - Agganci all'attualità }\end{array}$ \\
\hline
\end{tabular}




\begin{tabular}{|c|c|}
\hline $\begin{array}{l}\text { Area } 2 \text { - Relazione didattica } \\
\quad \text { Indicatori } \\
\text { - Clima d'aula } \\
\text { - Regole di comportamento } \\
\text { - Comunicazione in aula } \\
\text { - Corpo e corporeità }\end{array}$ & $\begin{array}{l}\text { - Riferimenti alle Indicazioni } \\
\text { Nazionali }\end{array}$ \\
\hline $\begin{array}{l}\text { Area } 3 \text { - Processi mediatori } \\
\text { Indicatori } \\
\text { - Area “gestione" del campo di } \\
\text { esperienza/ disciplina } \\
\text { - Area interventi mediatori per } \\
\text { l'apprendimento } \\
\text { - Area mediatori didattici } \\
\text { - Area relazione pedagogica in- } \\
\text { segnante-allievo }\end{array}$ & \\
\hline $\begin{array}{l}\text { Area } 4 \text { - Inclusione didattica } \\
\text { Indicatori } \\
\text { - Co-conduzione attività d'aula } \\
\text { - Metodologie inclusive } \\
\text { - Valori inclusivi } \\
\text { - “Tempi” inclusivi } \\
\text { - “Spazi” inclusivi }\end{array}$ & \\
\hline
\end{tabular}

Tab. 2 - Indice check list - Scheda di analisi video

A titolo esemplificativo si riporta la scheda di analisi relativa all'asse 2 "Agire studente":

\begin{tabular}{|l|l|}
\hline $\begin{array}{l}\text { Asse 2 - Agire studente } \\
\text { Area 5 - Il "fare" degli studenti }\end{array}$ \\
\hline Indicatori & Area commento \\
\hline T. Gestione "spazio" alunni & \\
\hline U. Gestione "tempo" alunni & \\
\hline V. Relazioni in aula & \\
\hline $\begin{array}{l}\text { W. Partecipazione degli stu- } \\
\text { denti }\end{array}$ \\
\hline
\end{tabular}

Tab. 3 - Scheda di analisi relativa all'asse 2 - "Agire studente” 


\begin{tabular}{|c|c|}
\hline \multicolumn{2}{|c|}{$\begin{array}{l}\text { Appendici: descrittori per area commento } \\
\text { Area } 5 \text { - II "fare" degli studenti }\end{array}$} \\
\hline Indicatori & Descrittori \\
\hline $\begin{array}{l}\text { T. Gestione } \\
\text { "spazio" } \\
\text { alunni }\end{array}$ & $\begin{array}{l}\text { T. } 1 \text { Gli studenti si muovono nello spazio aula } \\
\text { T. } 2 \text { Gli studenti sono seduti }\end{array}$ \\
\hline $\begin{array}{l}\text { U. Gestione } \\
\text { "tempo" } \\
\text { alunni }\end{array}$ & $\begin{array}{l}\text { U. } 1 \text { Gli studenti chiedono aiuto all'insegnante } \\
\text { U. } 2 \text { Gli studenti alzano la mano per intervenire } \\
\text { U. } 3 \text { Gli studenti richiedono chiarimenti sugli argomenti esposti dal do- } \\
\text { cente } \\
\text { U. } 4 \text { Gli studenti esprimono un contributo personale } \\
\text { U. } 5 \text { Gli studenti esprimono dissenso } \\
\text { U. } 6 \text { Gli studenti si confrontano con i compagni } \\
\text { U. } 7 \text { Gli studenti intervengono senza rispettare i turni di parola } \\
\text { U. } 8 \text { Gli studenti svolgono un'esercitazione richiesta } \\
\text { U. } 9 \text { Gli studenti interrompono l'attività, chiedendo di uscire } \\
\text { U. } 10 \text { Gli studenti prendono appunti } \\
\text { U. } 11 \text { Gli studenti leggono il libro di testo } \\
\text { U. } 11 \text { Gli studenti usano altri supporti offerti dall'insegnante } \\
\text { U. } 12 \text { Gli studenti usano supporti tecnologici (pc; i-phone; i-pad, altro) }\end{array}$ \\
\hline $\begin{array}{l}\text { V. Relazioni } \\
\text { in aula }\end{array}$ & $\begin{array}{l}\text { V. } 1 \text { Si osservano gesti amichevoli degli studenti verso l'insegnante } \\
\text { V. } 2 \text { Tra gli studenti c'è collaborazione e supporto vicendevole } \\
\text { V. } 3 \text { Gli studenti si rivolgono all'insegnante anche quando non sono diret- } \\
\text { tamente interpellati o interrogati } \\
\text { V. } 4 \text { Non si osservano comportamenti oppositivi da parte degli studenti } \\
\text { (rispondere in modo brusco, non seguire le consegne dell'insegnante) } \\
\text { V. } 5 \text { Non si osservano gesti amichevoli degli studenti verso l'insegnante } \\
\text { V. } 6 \text { Tra gli studenti non c'è collaborazione e supporto } \\
\text { V. } 7 \text { Gli studenti dimostrano di non saper rispettare le regole }\end{array}$ \\
\hline $\begin{array}{l}\text { W. Partecipa- } \\
\text { zione degli } \\
\text { studenti }\end{array}$ & $\begin{array}{l}\text { W. } 1 \text { Gli studenti seguono la lezione con molta attenzione } \\
\text { W. } 2 \text { Gli studenti partecipano attivamente alla lezione con domande di ap- } \\
\text { profondimento } \\
\text { W. } 3 \text { Gli studenti mostrano di impegnarsi nelle attività proposte } \\
\text { W. } 4 \text { Gli studenti seguono la lezione passivamente } \\
\text { W. } 5 \text { Gli studenti non partecipano alla lezione } \\
\text { W. } 6 \text { Gli studenti durante le attività non sono concentrati sul compito }\end{array}$ \\
\hline $\begin{array}{l}\text { Y. Risposte } \\
\text { degli studenti }\end{array}$ & $\begin{array}{l}\text { Y. } 1 \text { Gli studenti rispondono alle domande del docente in modo mnemo- } \\
\text { nico } \\
\text { Y. } 2 \text { Gli allievi rispondono alle domande del docente riportando le infor- } \\
\text { mazioni richieste } \\
\text { Y. } 3 \text { Gli allievi rispondono alle domande del docente mostrando di aver } \\
\text { compreso i concetti proposti nel corso della lezione } \\
\text { Y. } 4 \text { Gli allievi rispondono alle domande del docente operando collega- } \\
\text { menti e ampliamenti } \\
\text { Y. } 5 \text { Gli allievi rispondono alle domande del docente con appunti critici } \\
\text { sugli argomenti proposti }\end{array}$ \\
\hline
\end{tabular}

Tab. 4 - Appendice: descrittori per area commento (Area 5 - Il “fare” degli studenti) 


\section{Risultanze: la videovalutazione come dispositivo documentale per la valutazione autentica}

Attraverso la sperimentazione, le scuole hanno potuto analizzare i compiti autentici con il supporto delle schede precedentemente descritte del dispositivo di videovalutazione e strutturare schede di analisi del compito più efficaci, attraverso una scelta personalizzata e adattata degli indicatori di osservazione, come nell'esempio di seguito riportato (redatto dall'I.C. "Renato Moro" di Taranto):

\begin{tabular}{|c|c|c|c|c|}
\hline $\begin{array}{c}\text { TIPOLOGIA } \\
\text { DI COMPITO } \\
\text { AUTENTICO } \\
\text { DA } \\
\text { OSSERVARE } \\
\text { E VALUTARE }\end{array}$ & $\begin{array}{c}\text { DIMENSIONI DI } \\
\text { REALIZZAZIONE } \\
\text { DEL COMPITO } \\
\text { AUTENTICO DA } \\
\text { OSSERVARE E } \\
\text { VALUTARE }\end{array}$ & $\begin{array}{c}\text { COMPETENZE PROFILO } \\
\text { DELLO STUDENTE } \\
\text { (Modello MIUR per la Certifica- } \\
\text { zione delle competenze, CM 3) }\end{array}$ & $\begin{array}{c}\text { LIVELLI AT- } \\
\text { TESI DI } \\
\text { PADRONANZA }\end{array}$ & $\begin{array}{l}\text { VALUTAZIONE } \\
\text { DELLE } \\
\text { COMPETENZE } \\
\text { RILEVATE } \\
\text { ATTRAVERSO } \\
\text { OSSERVAZIONI } \\
\text { SISTEMATICHE }\end{array}$ \\
\hline \multirow[t]{3}{*}{$\begin{array}{c}\text { Presentazione } \\
\text { complessiva } \\
\text { dell'azione attra- } \\
\text { verso videoriprese } \\
\text { (da } 10 \text { a } 20 \text { minuti) } \\
\text { COMPITO UNI- } \\
\text { TARIO con con- } \\
\text { notazioni ascrivi- } \\
\text { bili al tipo “PER- } \\
\text { SONALE", vista } \\
\text { la spiccata eviden- } \\
\text { ziazione/valuta- } \\
\text { zione di compe- } \\
\text { tenze esistenziali e } \\
\text { trasversali }\end{array}$} & 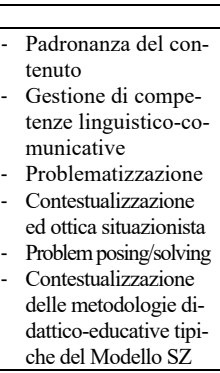 & $\begin{array}{l}\text { - Comunicazione nella madrelin- } \\
\text { gua o lingua di istruzione. } \\
\text { - Competenze digitali. } \\
\text { - Imparare ad imparare. } \\
\text { - Consapevolezza ed espressione } \\
\text { culturale. } \\
\text { - Spirito di iniziativa e imprendi- } \\
\text { torialità. } \\
\text { - Competenze sociali e civiche. }\end{array}$ & $\begin{array}{l}\text { 1) AVANZATO } \\
\text { 2) INTERMEDIO } \\
\text { 3) BASE } \\
\text { 4) INIZIALE }\end{array}$ & $\begin{array}{l}\text { 1. INTERAZIONE/ } \\
\text { PARTECIPAZIONE } \\
\text { 2. DIMENSIONE } \\
\text { COOPERATIVA } \\
\text { 3. AUTONOMIA E } \\
\text { FLESIBILITA' } \\
\text { 4. PROGETTAZIONE } \\
\text { E PIANIFICAZIONE } \\
\text { 5. VALUTAZIONE } \\
\text { CRITICA } \\
\text { 6. APPORTO CREA- } \\
\text { TIVO (originalità della } \\
\text { proposta progettuale) }\end{array}$ \\
\hline & \multicolumn{4}{|c|}{ INDICATORI DI ANALISI DELLA COMPETENZA } \\
\hline & $\begin{array}{l}\text { 1) Recuperare ed utiliz- } \\
\text { zare conoscenze e compe- } \\
\text { tenze pluridisciplinari } \\
\text { 2) Gestire la comunica- } \\
\text { zione linguistica e l'elabo- } \\
\text { razione contenutistica, at- } \\
\text { traverso esercizio e conso- } \\
\text { lidamento di competenze } \\
\text { esistenziali e trasversali. } \\
\text { 3) Avviare la produzione } \\
\text { conoscitiva e l'attivazione } \\
\text { di competenze problema- } \\
\text { ticamente } \\
\text { 4) Contestualizzare aspetti } \\
\text { conoscitivi e competenze } \\
\text { 5) Produrre conoscenze ed } \\
\text { esercitare competenze in } \\
\text { situazione } \\
\text { 6) Esercitare concreta- } \\
\text { mente il criterio della Re- } \\
\text { sponsabilità, per un'edu- } \\
\text { cazione alla partecipa- } \\
\text { zione democratica } \\
\text { 7) Attivare capacità deci- } \\
\text { sionale ed abilità organiz- } \\
\text { zative } \\
\text { 8) Indicare soluzioni ge- } \\
\text { stionali } \\
\text { 9) Utilizzare sistematica- } \\
\text { mente procedure e strate- } \\
\text { gie innovative }\end{array}$ & $\begin{array}{l}\text { 1) Ha una padronanza della lingua ita- } \\
\text { liana tale da consentirgli di compren- } \\
\text { dere enunciati e testi di una certa com- } \\
\text { plessita, di esprimere le proprie idee, di } \\
\text { adottare un registro linguistico appro- } \\
\text { priato alle diverse situazioni } \\
\text { 2) Usa con consapevolezza le tecnologie } \\
\text { della comunicazione per ricercare e ana- } \\
\text { lizzare dati ed informazioni, per distin- } \\
\text { guere informazioni attendibili da quelle } \\
\text { che necessitano di approfondimento, di } \\
\text { controllo e di verifica e per interagire } \\
\text { con soggetti diversi nel mondo } \\
\text { 3) Possiede un patrimonio organico di } \\
\text { conoscenze e nozioni di base ed è allo } \\
\text { stesso tempo capace di ricercare e di } \\
\text { procurarsi velocemente nuove informa- } \\
\text { zioni ed impegnarsi in nuovi apprendi- } \\
\text { menti anche in modo autonomo } \\
\text { 4) Utilizza gli strumenti di conoscenza } \\
\text { per comprendere se stesso e gli altri, } \\
\text { per riconoscere ed apprezzare le di- } \\
\text { verse identità, le tradizioni culturali e } \\
\text { religiose, in un'ottica di dialogo e di ri- } \\
\text { spetto reciproco. Interpreta i sistemi } \\
\text { simbolici e culturali della società } \\
\text { 5) Dimostra originalità e spirito di inizia- } \\
\text { tiva. Si assume le proprie responsabilità, } \\
\text { chiede aiuto quando si trova in difficoltà̀ } \\
\text { e sa fornire aiuto a chi lo chiede. Ė di- } \\
\text { sposto ad analizzare se stesso e a misu- } \\
\text { rarsi con le novità e gli imprevisti } \\
\text { 6) Rispetta le regole condivise, colla- } \\
\text { bora con gli altri per la costruzione del } \\
\text { bene comune esprimendo le proprie } \\
\text { personali opinioni e sensibilità }\end{array}$ & $\begin{array}{l}\text { 1) Livello soddisfa- } \\
\text { cente } \\
\text { 2) Livello adeguato } \\
\text { 3) Livello accettabile } \\
\text { 4) Livello non iniziale }\end{array}$ & $\begin{array}{l}\text { Individuare, verificare } \\
\text { e valutare } \\
\text { 1. Capacità relazionale } \\
\text { e di integrazione di } \\
\text { gruppo } \\
\text { 2. Livello di partecipa- } \\
\text { zione e di intervento } \\
\text { 3. Disponibilità alla } \\
\text { collaborazione e condi- } \\
\text { visione } \\
\text { 4. Riflessione e ge- } \\
\text { stione autonoma di sa- } \\
\text { peri, strumenti, espe- } \\
\text { rienze } \\
\text { 5. Apertura alla flessi- } \\
\text { bilità delle posizioni } \\
\text { 6. Capacità di organiz- } \\
\text { zazione/gestione e } \\
\text { coordinamento } \\
\text { 7. Capacità di valuta- } \\
\text { zione critica } \\
\text { 8. Propensione all'ori- } \\
\text { ginalità/creatività della } \\
\text { proposta e delle inizia- } \\
\text { tive correlate }\end{array}$ \\
\hline
\end{tabular}

Tab. 5 - Esempio di scheda di analisi del compito autentico 
Si riporta di seguito un estratto di scrittura post-analisi video che un docente ha postato nell'ambiente condiviso e-learning, in cui è esplicitata la funzione della videoanalisi nella compilazione della rubrica e nei processi valutativi e autovalutativi, pensati in ottica formativa e autentica (Wiggins, 1993):

"Rivedere il filmato consente, al docente, una più puntuale compilazione della rubrica valutativa elaborata per il compito perché costituisce una "memoria" costantemente fruibile per la valutazione autentica. L'osservazione delle prestazioni degli alunni aiuta a stabilire il livello raggiunto nei singoli descrittori di competenza che riguardano la parte esecutiva del compito ovvero quella filmata. Usufruire di questo strumento significa essere certi di non tralasciare o dimenticare i passaggi salienti della prestazione autentica. Gli alunni hanno, da parte loro, molto gradito la novità di essere filmati ed hanno profuso un maggiore impegno nel compito perché hanno percepito l'utilizzo della videoripresa come un passaggio importante e accurato della loro valutazione e, rivedendo il filmato, sono stati molto critici nel giudicare la loro performance e si sono quindi autovalutati”.

La sperimentazione ha mostrato come la videoanalisi delle pratiche educative costituisca un dispositivo documentale efficace che sfrutta le potenzialità delle ICT (Galliani, 2014) nella descrizione, archiviazione, rappresentazione, produzione e interpretazione degli eventi educativi di natura sociale, in quanto capaci di modificare i ruoli dei soggetti implicati nel processo conoscitivo, le dinamiche di interazione-comunicazione, le possibilità di accesso al sapere, la flessibilità nella costruzione di ambienti di apprendimento.

L'efficacia di un dispositivo documentale videovalutativo è centrale in quanto, come sostiene Vinci (2021), consente di raccogliere e documentare evidenze e di ottenere dati 'affidabili', basati su indicatori, ossia su elementi osservabili, rilevabili, che consentono di operativizzare concetti teorici e di rinviare a qualcosa che rivela la presenza di un determinato fenomeno, ma rimandano ad un significato che va letto attraverso un processo interpretativo personale: gli indicatori vengono definiti come "concetti "specifici" traducibili in termini osservativi che sono legati ai concetti "generali" da quello che viene definito un rapporto di indicazione o rappresentazione semantica'. Affinché l'insegnante possa 'leggere' gli indicatori e attribuirvi un senso attraverso un processo interpretativo - dando senso all'azione - è opportuno scegliere e costruire strumenti adeguati con cui misurare l'apprendimento, stabilire criteri intersoggettivi di qualità per formulare ed esprimere un giudizio valutativo intorno a 
ciò che è stato misurato, costruire un sistema documentale affidabile di gestione del dato (Perla, 2019), in grado di supportare nella raccolta delle informazioni e dei dati utili per valutare un certo oggetto.

«Documentare con competenza permette all'insegnante di raccogliere evidenze a supporto del proprio operato, di saper interpretare i dati per individuare priorità di azioni migliorative; di riuscire a monitorare l'andamento dei processi mobilitati rendendone conto alla comunità degli stakeholders con la trasparenza necessaria a esternalizzare il valore di una Scuola e delle azioni dei suoi operatori: dirigenti, personale docente e amministrativo. Solo una valutazione nella sua funzione valorizzante (...) può assolvere alla delicata funzione di visibilizzare il livello di professionalità espresso in una Scuola: professionalità che, in assenza di strumenti valutativi/valorizzanti, continuerà a restare oscura e mai veramente riconosciuta» (Perla, 2019, p. 31).

La videovalutazione consente all'insegnante di raccogliere informazioni a partire dalle quali condurre processi valutativi e autovalutativi in ottica formativa, rendendo visibile l'apprendimento e consentendo di mantenere una memoria di quanto detto e fatto, di riguardarsi e di avere informazioni sul proprio modo di imparare. La videovalutazione rafforza la dimensione formativa della valutazione, che dovrebbe essere parte integrante del processo di insegnamento-apprendimento e generare feedback che possono essere utilizzati dagli studenti, per migliorare l'apprendimento, e dagli insegnanti, per la regolazione delle loro pratiche in accordo con le esigenze dei loro studenti (Nicol, Macfarlane-Dick, 2004).

Sebbene consapevoli che la sperimentazione costituisca solo un case study e che non è possibile effettuare delle generalizzazioni in assenza di ulteriori evidenze, l'esperienza condivisa con i docenti suggerisce come la videovalutazione possa supportare, nella valutazione dell'agire competente dello studente, l'individuazione dei livelli in cui si esprime la competenza (Castoldi, 2016): essa può supportare, quindi, la costruzione della rubrica valutativa e l'espressione, da parte del docente, del giudizio finale per livelli.

Quando coinvolge anche gli studenti, la videovalutazione può supportare lo sviluppo di processi autovalutativi e di competenze metacognitive, l'autoregolazione dell'apprendimento, la consapevolezza delle proprie strategie cognitive. Ma non solo: rappresenta indubbiamente anche un valido supporto nella 'restituzione' agli alunni e ai genitori della valutazione in itinere. 


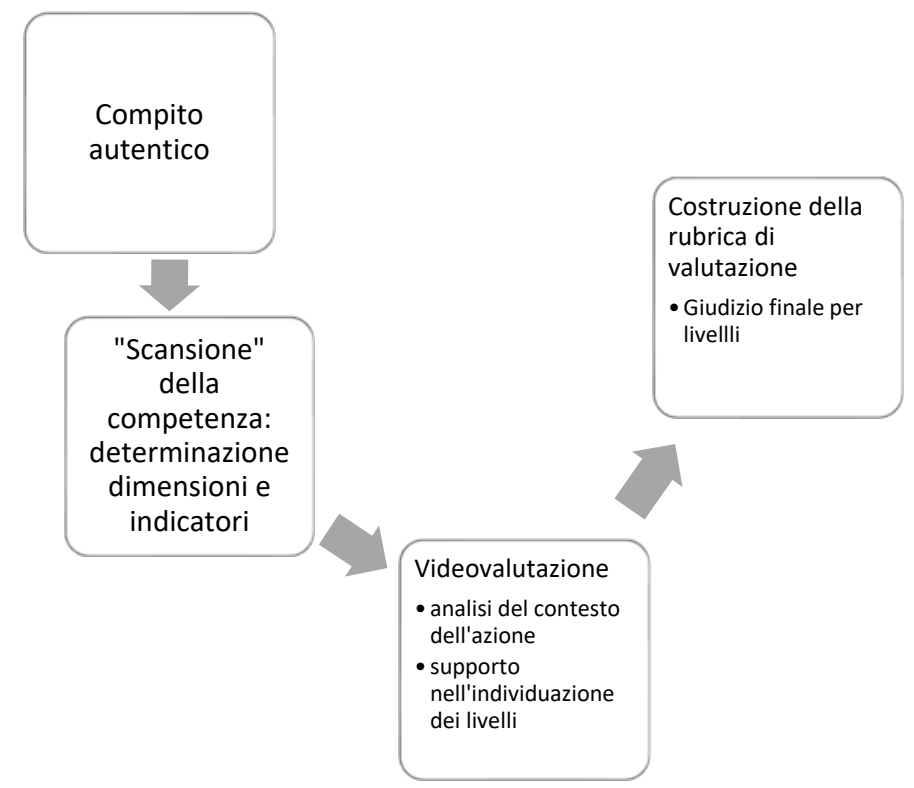

Fig. 2 - Sequenze della valutazione delle competenze supportata dalla videovalutazione

Da ultimo: i dati della ricerca, restituiti agli insegnanti entro un setting riflessivo e autovalutativo, hanno consentito di assumere una postura di analisi delle proprie pratiche didattiche in situazione.

Più specificatamente il dispositivo di videovalutazione sperimentato nel corso di questa ricerca ha consentito alle/ai docenti di oggettivare la propria azione didattica, requisito per impostare un'attività sistematica di riflessione. In questa direzione l'attività riflessiva sollecitata nelle/nei docenti, attraverso l'uso del dispositivo proposto, ha avuto l'obiettivo di accompagnare le/gli insegnanti a riconsiderare criticamente il proprio l'agire, sollecitando lo sviluppo e il consolidamento della propria professionalità.

La ricerca ha consentito dunque di promuovere negli insegnanti - attraverso l'uso sistematico di un dispositivo di videovalutazione - un habitus di riflessione sul proprio "fare scuola" e, più specificamente, sulle proprie strategie valutative, sul modo in cui si gestisce la mediazione tra soggetti in apprendimento, contenuti disciplinari, metodologie, strategie e dispositivi adottati al fine di contribuire in maniera consapevole alla stesura di piani di miglioramento.

E non solo. Il percorso intrapreso ha schiuso prospettive interessanti per implementare nelle scuole una "cultura" della documentazione non "schiacciata" sul versante burocratico-amministrativo ma finalizzata a supportare processi autovalutativi. A questo proposito occorre evidenziare che promuovere percorsi 
di autoanalisi e autovalutazione implica il rinnovamento delle competenze professionali dei docenti e in particolar modo della competenza documentativa (Perla, 2019). Da ciò la necessità di progettare dispositivi metodologici specifici che possano sostenere i docenti nelle azioni di valutazione e auto-valutazione, oltre che di far avanzare la ricerca sui modelli per lo sviluppo professionale dei docenti.

\section{Riferimenti bibliografici}

Ackermans, K., Rusman, E., Brand-Gruwel, S., Specht, M. (2017). A First Step Towards Synthesising Rubrics and Video for the Formative Assessment of Complex Skills. Technology Enhanced Assessment: 19th International Conference (Vol. 653, pp. 1-10). Springer, CCIS, vol. 653.

Allen, D.W. (1966). Micro-teaching is a scaled down teaching encounter in class size and class time. The High School Journal, 49(8), 355-362.

Altet, M. (2002). L'analyse plurielle de la pratique enseignante, une démarche de Recherche. Revue Française de Pédagogie, 138, 85-93.

Altet, M. (2003). La ricerca sulle pratiche d'insegnamento in Francia. Brescia: La Scuola.

Castoldi, M. (2016). Valutare e certificare le competenze. Roma: Carocci.

Cescato S., Bove C., Braga P. (2015). Video, formazione e consapevolezza. Intrecci metodologici.Form@re,15(2),61-74.

Damiano, E. (2013). La mediazione didattica. Per una teoria dell'insegnamento. Milano: FrancoAngeli.

Franchini, E., Salvisberg, M., Sbaragli, S. (2016). Riflessioni sulla valutazione formativa tramite l'uso di video. Linee guida per formatori. Locarno: SUPSI - Dipartimento formazione e apprendimento.

Galliani, L. (2014). Per una ontologia della valutazione educativa. In L. Galliani, A. Notti (Eds.), Valutazione educativa. Lecce: Pensa MultiMedia.

Galliani, L. (2015). L'agire valutativo. Manuale per docenti e formatori. Brescia: La Scuola.

Grion, V., Aquario, D., Restiglian, E. (2019). Valutare nella scuola e nei contesti educativi. Padova: CLEUP.

Henderson, M., Phillips, M. (2015). Video-based feedback on student assessment: scarily personal. Australasian Journal of Educational Technology, 31(1), 51-66.

Herrington, J., Reeves, T.C., Oliver, R. (2014). Authentic Learning Environments. In J.M. Spector, M.D. Merrill, J. Elen, M.J. Bishop (Eds.). Handbook of Research on Educational Communications and Technology. New York: Springer-Verlag.

Mangione, G.R., Rosa, A. (2017). Professional vision e il peer to peer nel percorso Neoassunti. L'uso del video per l'analisi della pratica del docente in classe. Form@re, 17(1), 120-143.

Nicol, D., Macfarlane-Dick, D. (2004). Rethinking formative assessment in HE: a theoretical model and seven principles of good feedback practice. In: C. Juwah, et al. 
(Eds.) (2004). Enhancing student learning though effective formative feedback. York: The Higher Education Academy.

Orme, M. (1966). The effects of modeling and feedback variables on the acquisition of a complex teaching strategy. (Unpublished doctoral dissertation). Stanford, California: Stanford University.

Perla, L. (Eds.) (2019). Valutare per valorizzare. La documentazione per il miglioramento di scuola, insegnanti, studenti. Brescia: Morcelliana.

Perla, L., Schiavone, N. (2014). Quels dispositifs de documentation de l'implicite dans la formation des enseignants? In M. Frisch (ed.), Le réseau IDEKI. Objets de recherche d'éducation et de formation émergents, problématisés, mis en tension, réélaborés (pp. 23-42). Paris: L'Harmattan.

Perla, L., Schiavone, N. (2017). Video-Analysis and Self-Assessment in Teaching Work. In P.G., Rossi, L., Fedeli (Eds.). Integrating Video into Pre-Service and InService Teacher Training (pp. 191-209). Hersey, PA: IGI Global.

Rossi, P.G., Fedeli, L. (Eds.). Integrating Video into Pre-Service and In-Service Teacher Training. Hersey, PA: IGI Global.

Santagata, R., Guarino, J. (2011). Using video to teach future teachers to learn from teaching. Zdm, 43(1), 133-145.

Vinci, V. (2021). La documentazione per la valutazione. Nuova Secondaria Ricerca, 9 - Dossier, pp. 445-457.

Wiggins, G. (1993), Assessing student performance: Exploring the purpose and limits of testing. San Francisco, CA: Jossey-Bass. 